\title{
Silent Intracerebral Hemorrhage in Patients Randomized to Stenting or Endarterectomy for Symptomatic Carotid Stenosis
}

\author{
Mandy D. Müller, ${ }^{\mathrm{a}}$ Lisa M. Jongen, ${ }^{\mathrm{b}}$ Aysun Altinbas, ${ }^{\mathrm{c}}$ Kristine A. Blackham, ${ }^{\mathrm{d}}$ Paul J. Nederkoorn, \\ Sumaira Macdonald, ${ }^{\mathrm{f}}$ Rolf Jäger, ${ }^{\mathrm{g}}$ Thomas Wolff, ${ }^{\mathrm{h}}$ Philippe A. Lyrer, ${ }^{\mathrm{a}}$ L. Jaap Kappelle, ${ }^{\mathrm{i}}$ \\ Stephan G. Wetzel, ${ }^{\mathrm{j}}$ Toby Richards, ${ }^{\mathrm{k}}$ Jeroen Hendrikse, ${ }^{\mathrm{b}}$ Gert J. de Borst, ${ }^{1}$ H. Bart van der Worp, ${ }^{\mathrm{i}}$ \\ Stefan T. Engelter, ${ }^{\mathrm{a}, \mathrm{m}}$ David J. Werring, ${ }^{\mathrm{n}}$ Martin M. Brown, ${ }^{\mathrm{n}}$ Leo H. Bonati, ${ }^{\mathrm{a}, \mathrm{n}}$ on behalf of the ICSS-MRI \\ Study Investigators \\ ${ }^{a}$ Department of Neurology and Stroke Center, University Hospital Basel, University of Basel, Basel, Switzerland \\ ${ }^{b}$ Department of Radiology, University Medical Center Utrecht, University The Netherlands, Utrecht, the Netherlands \\ 'Department of Pediatric Neurology, Leiden University Medical Center, Leiden University, Leiden, the Netherlands \\ ${ }^{d}$ Department of Diagnostic and Interventional Neuroradiology, University Hospital Basel, University of Basel, Basel, Switzerland \\ 'Department of Neurology, Academic Medical Center, Amsterdam, the Netherlands \\ fDepartment of Radiology, Freeman Hospital, Newcastle upon Tyne, UK \\ ${ }^{9}$ Academic Neuroradiological Unit, Department of Brain Repair and Rehabilitation, UCL Queen Square Institute of Neurology, London, UK \\ hDepartment of Vascular Surgery, University Hospital Basel, University of Basel, Basel, Switzerland \\ 'Department of Neurology and Neurosurgery, Rudolf Magnus Institute of Neuroscience, University Medical Center Utrecht, Utrecht University, \\ Utrecht, the Netherlands \\ institute of Neuroradiology, Clinic Hirslanden, Zurich, Switzerland \\ ${ }^{k}$ Division of Surgery and Interventional Sciences, University College London, London, UK \\ 'Department of Vascular Surgery, University Medical Center Utrecht, Utrecht University, Utrecht, the Netherlands \\ ${ }^{m}$ Neurorehabilitation Unit, University Center for Medicine of Aging and Rehabilitation Basel, Felix Platter Hospital, University of Basel, Basel, \\ Switzerland \\ nDepartment of Brain Repair and Rehabilitation, UCL Queen Square Institute of Neurology, London, UK
}

\section{Dear Sir:}

Carotid artery stenting (CAS) is a less invasive alternative to carotid endarterectomy (CEA) for treatment of symptomatic carotid stenosis. The risk of peri-procedural cerebral ischemia is greater with CAS than with CEA. ${ }^{1}$ However, evidence on the occurrence of new hemorrhagic brain lesions after CAS or CEA is sparse. We aimed to assess the frequency of clinically silent brain hemorrhage on magnetic resonance imaging (MRI) after CAS and CEA in the MRI substudy of the International Carotid Stenting Study (ICSS), a registered clinical trial (RCTN25337470).

In ICSS, patients with symptomatic carotid stenosis were randomized in a 1:1 ratio to CAS or CEA. In the ICSS-MRI substudy, patients were scanned with multi-modal MRI 1 to 7 days before, 1 to 3 days after and $30 \pm 3$ days after revascularization, in- cluding gradient-recalled echo (GRE) T2* ${ }^{*}$-weighted MRI. The primary outcome of the present study was the presence of at least one new hemorrhagic brain lesion on the 30-day scan, defined by any new signal hypointensity within the brain parenchyma on GRE T2* which was not present before treatment. Small, rounded hypointense lesions measuring $\leq 10 \mathrm{~mm}$ in diameter were classified as cerebral microbleeds (CMBs). ${ }^{3}$ New hemorrhagic lesions on the 1-month follow-up scan occurring at the site of ischemic brain lesions seen on fluid-attenuated inversion recovery sequences before treatment, or at the site of new diffusion-weighted imaging lesions in the scan done 1 to 3 days after treatment, were classified as hemorrhagic transformations of ischemic infarcts, and were not counted as part of the primary outcome.

Details on the CAS and CEA procedures have been described 
Table 1. Baseline characteristics, radiological, and clinical outcome events

\begin{tabular}{|c|c|c|c|}
\hline Characteristic & CAS $(n=84)$ & CEA $(n=78)$ & Total $(n=162$ \\
\hline Age (yr) & $70.4 \pm 8.9$ & $69.4 \pm 8.5$ & $69.9 \pm 8.7$ \\
\hline Male sex & $65(77.4)$ & $53(67.9)$ & $118(72.8)$ \\
\hline \multicolumn{4}{|l|}{ Vascular risk factors } \\
\hline History of hypertension & $57(68.0)$ & $56(72.0)$ & $113(70.0)$ \\
\hline History of diabetes & $18(21.0)$ & $19(24.0)$ & $37(23.0)$ \\
\hline History of hypercholesterolemia & $54(64.0)$ & $54(69.0)$ & $108(67.0)$ \\
\hline Smoking (past or present) & $66(79.0)$ & $54(69.0)$ & $120(74.0)$ \\
\hline Coronary heart disease & $15(18.0)$ & $20(26.0)$ & $35(22.0)$ \\
\hline Peripheral artery disease & $11(13.0)$ & $10(13.0)$ & $21(13.0)$ \\
\hline \multicolumn{4}{|l|}{ Blood pressure at randomization $(\mathrm{mm} \mathrm{Hg})$} \\
\hline Systolic & $158.6 \pm 28.3$ & $158.2 \pm 25.1$ & $158.4 \pm 26.8$ \\
\hline Diastolic & $81.9 \pm 14.0$ & $81.9 \pm 11.9$ & $81 \cdot 9 \pm 13.0$ \\
\hline Mean & $107.5 \pm 17.2$ & $107.4 \pm 14.9$ & $107.4 \pm 16.1$ \\
\hline \multicolumn{4}{|l|}{ Blood pressure at hospital discharge $(\mathrm{mm} \mathrm{Hg})$} \\
\hline Systolic & $135.1 \pm 19.8$ & $134.7 \pm 17.1$ & $134.9 \pm 18.4$ \\
\hline Diastolic & $71.6 \pm 12.8$ & $72.5 \pm 10.8$ & $72.1 \pm 11.8$ \\
\hline Mean & $92.8 \pm 13.0$ & $93.3 \pm 11.3$ & $93.0 \pm 12.1$ \\
\hline \multicolumn{4}{|l|}{ Blood pressure at 1 month follow-up $(\mathrm{mm} \mathrm{Hg})$} \\
\hline Systolic & $157.9 \pm 21.7$ & $152.4 \pm 23.5$ & $155.2 \pm 22.7$ \\
\hline Diastolic & $83.6 \pm 11.8$ & $81.5 \pm 10.8$ & $82.6 \pm 11.4$ \\
\hline Mean & $108.4 \pm 13.5$ & $105.1 \pm 13.5$ & $106.8 \pm 13.5$ \\
\hline \multicolumn{4}{|l|}{ Most recent ipsilateral event ${ }^{*}$} \\
\hline Amaurosis fugax & $21(25.0)$ & $14(18.0)$ & $35(22.0)$ \\
\hline Retinal stroke & $5(6.0)$ & $1(1.0)$ & $6(4.0)$ \\
\hline Transient ischemic attack & $24(29.0)$ & $30(40.0)$ & $54(34.0)$ \\
\hline Hemispheric stroke & $34(41.0)$ & $31(41.0)$ & $65(41.0)$ \\
\hline \multicolumn{4}{|l|}{ Score on modified Rankin Scale at randomization } \\
\hline 0 & $38(45.0)$ & $28(36.0)$ & $66(41.0)$ \\
\hline 1 & $19(23.0)$ & $19(24.0)$ & $38(24.0)$ \\
\hline 2 & $23(27.0)$ & $21(27.0)$ & $44(27.0)$ \\
\hline 3 & $4(5.0)$ & $8(10.0)$ & $12(7.0)$ \\
\hline 4 & $0(0.0)$ & $2(3.0)$ & $2(1.0)$ \\
\hline \multicolumn{4}{|l|}{ Degree of ipsilateral carotid stenosis at randomization ${ }^{+}$} \\
\hline Moderate (50\%-69\%) & $13(16.0)$ & $7(9.0)$ & $20(13.0)$ \\
\hline Severe (70\%-99\%) & $71(85.0)$ & $71(91.0)$ & $142(88.0)$ \\
\hline Contralateral severe carotid stenosis or occlusion & $4(5.0)$ & $3(4.0)$ & $7(4.0)$ \\
\hline \multicolumn{4}{|l|}{ Baseline MRI characteristics ${ }^{\ddagger}$} \\
\hline Presence of ischemic lesion on DWI before treatment & $32(38.0)$ & $29(37.0)$ & $61(38.0)$ \\
\hline Presence of CMB on GRE T2* before treatment & $16(19.0)$ & $12(15.0)$ & $28(17.0)$ \\
\hline Total ARWMC score & $4(2-7)$ & $4(2-8)$ & $4(2-7)$ \\
\hline \multicolumn{4}{|l|}{ Radiological outcome events on 1-month MRI } \\
\hline New hemorrhagic brain lesions & $5(6.0)$ & $5(6.4)$ & $10(6.2)$ \\
\hline Ipsilateral carotid territory & 4 & 4 & 8 \\
\hline Contralateral carotid or vertebrobasilar territory & 1 & 1 & 1 \\
\hline
\end{tabular}


Table 1. Continued

\begin{tabular}{|c|c|c|c|}
\hline Characteristic & CAS $(n=84)$ & CEA $(n=78)$ & Total $(n=162)$ \\
\hline \multicolumn{4}{|c|}{ Clinical outcome events } \\
\hline Death & 0 & 0 & 0 \\
\hline Any stroke & $7(8.3)$ & $3(3.8)$ & $10(6.2)$ \\
\hline Ischemic & 7 & 3 & 10 \\
\hline Hemorrhagic & 0 & 0 & 0 \\
\hline
\end{tabular}

Values are presented as mean \pm standard deviation, number $(\%)$, or median (interquartile range).

CAS, carotid artery stenting; CEA, carotid endarterectomy; MRI, magnetic resonance imaging; DWI, diffusion-weighted imaging; CMB, cerebral microbleed; GRE, gradient-recalled echo; ARWMC, age-related white matter changes.

*Type of qualifying event was not available in two patients in the CEA arm; ${ }^{+}$Degree of stenosis according to North American Symptomatic Carotid Endarterectomy Trial (NASCET) method; ${ }^{\ddagger}$ On pre-treatment scan done within International Carotid Stenting Study (ICSS)-MRI Study.

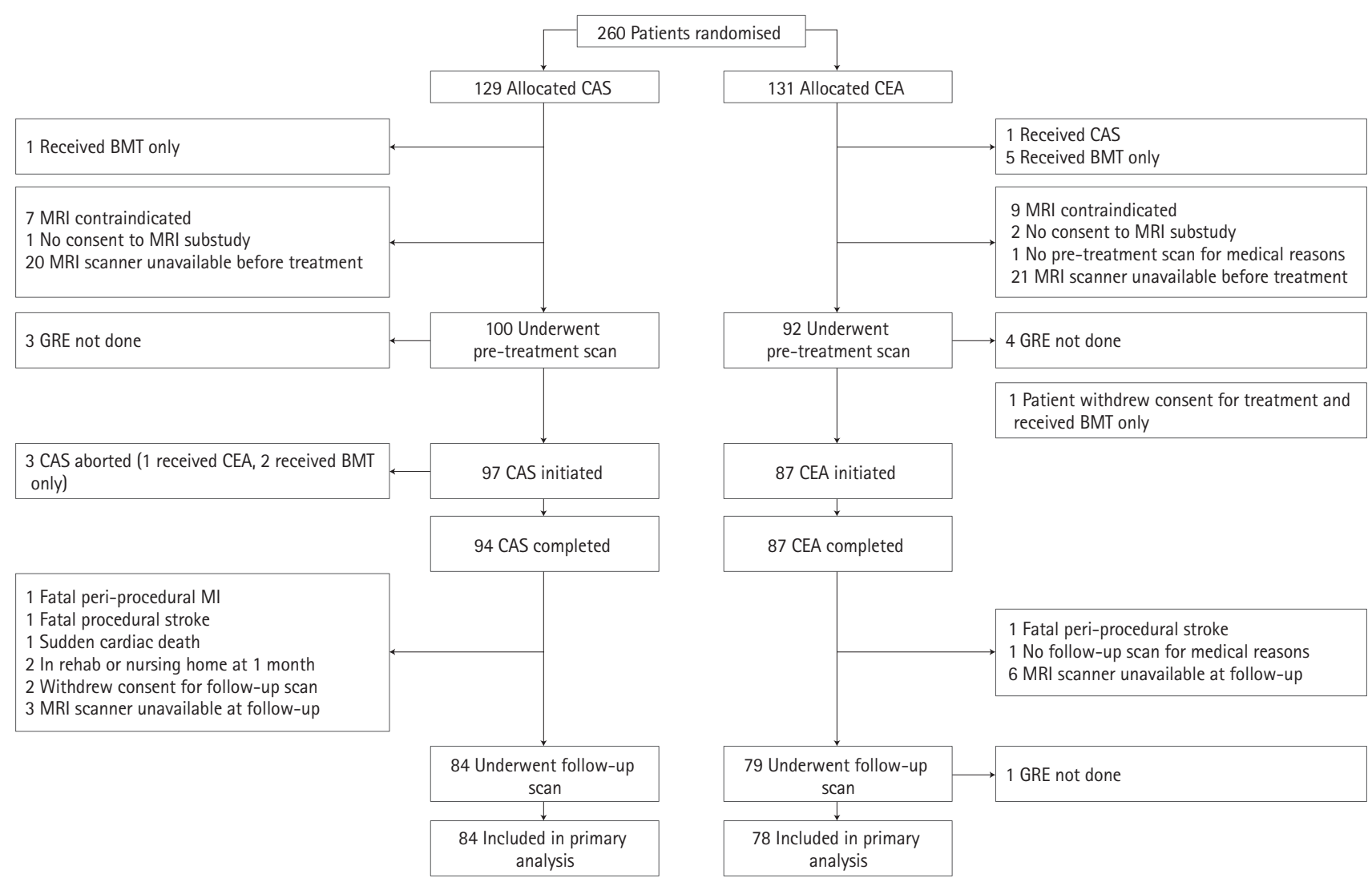

Figure 1. Study flow chart. Inclusion and reasons for exclusion of patients from analysis. CAS, carotid artery stenting; CEA, carotid endarterectomy; BMT, best medical therapy; MRI, magnetic resonance imaging; GRE, gradient-recalled echo; MI, myocardial infarction.

in detail before. ${ }^{2}$ In short, premedication for CAS was discretionary, although the combination of aspirin and clopidogrel was recommended. Use of heparin during the procedure was mandatory. All CEA patients received antiplatelet therapy before and after the procedure. Assessment of MRI scans was done through consensus reading by two experienced readers who were blinded to the allocated treatment and clinical outcome. In case of disagreement between the two, a third reviewer made the final decision.

We analyzed all patients who received the randomly allocat- ed treatment (CAS or CEA) and both the pre-treatment and 30-day MRI scans including GRE T2* sequences in sufficient quality. We compared the proportion of patients with the primary outcome between the CAS and the CEA group using Fisher's exact test. Binary logistic regression analysis was performed to study the association between each of the clinical and radiological baseline variables (Table 1) and the primary outcome in both treatment groups combined.

From 2005 to 2009, 260 patients were randomized at five ICSS-MRI substudy centers (CAS, $n=129 ;$ CEA, $n=131$ ). Our 
study population included 162 patients (CAS, $n=84$; CEA, $n=78$ ) who underwent treatment as randomly allocated and had complete MRI scans done before and 30 days after treatment. Patient flow and reasons for exclusion from the present analysis are detailed in Figure 1. Patients were well matched in baseline characteristics (Table 1). Thirty-one patients were studied in 3 Tesla scanners (CAS, $n=15 ; C E A, n=16$ ) and 131 in 1.5 Tesla scanners (CAS, $n=69 ;$; CEA, $n=62$ ).

Seven patients in the CAS group (8.3\%) and three patients in the CEA group (3.8\%) suffered an ischemic stroke. All pre-existing hemorrhagic brain lesions seen on the pre-treatment scan were still visible on the 1-month follow-up scan. No clinically manifest hemorrhagic strokes occurred. Hemorrhagic brain lesions, exclusively in the form of a single $\mathrm{CMB}$, occurred in five patients in the CAS group (6.0\%) and in five patients in the CEA group $(6.4 \%, P=0.88)$. CMBs were located in the territory supplied by the treated carotid artery in eight patients and in the contralateral carotid territory in two patients. In the CAS group, two CMBs were located in deep structures (basal ganglia), two in the frontal lobe, and one in the temporal lobe. In the CEA group, two CMBs were located in deep structures (external capsule and deep/periventricular white matter), two in the frontal lobe, and one in the temporal lobe. None of the patients with $\mathrm{CMBs}$ had symptoms attributable to the lesion or cerebral hyperperfusion syndrome (CHS). CMBs were not associated with any patient baseline characteristics including presence of CMBs before treatment, or baseline severity of white matter changes.

Our study has limitations. Due to the small number of patients exhibiting new hemorrhagic brain lesions on MRI after carotid revascularization, we are not able to draw any meaningful conclusions on the association between hemorrhagic brain lesions and $\mathrm{CHS}$, nor any of the assessed baseline risk factors including pre-existing CMBs. Second, no information was available on blood pressure values directly after CAS or CEA, or within a few days thereafter. In addition, only about one fifth of our patients were imaged on 3 Tesla scanners, and susceptibility weighted imaging sequences were not done. Therefore, we might have underestimated the true frequency of CMBs to some degree.

In conclusion, we found that hemorrhagic brain lesions after carotid revascularization are rare, do not differ between CAS and CEA, and in our study exclusively occurred in the form of a single $\mathrm{CMB}$, predominantly located in the territory supplied by the treated carotid artery.

\section{References}

1. Bonati LH, Jongen LM, Haller S, Flach HZ, Dobson J, Nederkoorn PJ, et al. New ischaemic brain lesions on MRI after stenting or endarterectomy for symptomatic carotid stenosis: a substudy of the international carotid stenting study (ICSS). Lancet Neurol 2010;9:353-362.

2. International Carotid Stenting Study investigators, Ederle J, Dobson J, Featherstone RL, Bonati LH, van der Worp HB, et al. Carotid artery stenting compared with endarterectomy in patients with symptomatic carotid stenosis (international carotid stenting study): an interim analysis of a randomised controlled trial. Lancet 2010;375:985-997.

3. Gregoire $S M$, Chaudhary UJ, Brown MM, Yousry TA, Kallis $C$, Jäger HR, et al. The Microbleed Anatomical Rating Scale (MARS): reliability of a tool to map brain microbleeds. Neurology 2009;73:1759-1766.

Correspondence: Leo H. Bonati

Department of Neurology and Stroke Center, University Hospital Basel, University of Basel, Petersgraben 4, CH-4031 Basel, Switzerland

Tel: +41-61-2652525

Fax: +41-61-2654100

E-mail: leo.bonati@usb.ch

Received: October 1, 2018

Revised: January 15, 2019

Accepted: January 15, 2019

The authors have no financial conflicts of interest. 